\title{
The unifying role of topology
}

In January 1851, using a two-metre-long pendulum hanging from the ceiling of his basement, the French physicist Léon Foucault demonstrated a surprising phenomenon - the slow but systematic clockwise rotation of the pendulum's oscillation plane. Scientists put on a repeat performance one month later at the Pantheon in Paris, this time with a pendulum ball of $28 \mathrm{~kg}$ suspended from a steel wire of $67 \mathrm{~m}$. The effect, of course, can easily be explained through the action of the Coriolis force in the Earth's non-inertial rotating frame, a perspective established by Gaspard-Gustave de Coriolis only two decades earlier.

Yet there is a more elegant perspective on Foucault's pendulum. Consider both the pendulum and Earth from a frame fixed on the Earth, but not rotating. We see the Earth's rotation carry the swinging pendulum along a circular line of latitude - in the case of the Paris pendulum, a circle at roughly $48.6^{\circ} \mathrm{N}$. At each point on its journey, the pendulum trajectory lies in a tangent plane to Earth's surface at that point, and inertia simply translates the line of the pendulum's horizontal motion parallel to itself in space, slightly further to the East. Yet after one period of oscillation, the Earth has rotated very slightly, and so the pendulum trajectory, though barely changed, now no longer belongs to the same tangent plane, but to the plane for another point just further along the circle of latitude.

This observation is surprisingly consequential. As the pendulum remains fixed to the Earth, its line in the new tangent plane can be found by projecting the parallel line down onto that new plane. In so doing, one finds that the line is not the same, but has rotated by a small angle, depending on the latitude. The result reveals that after a full day of oscillation, completing the circular course, the pendulum now makes a deflection angle $\varphi=2 \pi(1-\sin \theta)$ with its initial orientation, with $\theta$ the latitude. This is of course identical to a change caused by the Coriolis force, but emerges here from consideration of the so-called 'parallel transport' of the vector field representing the motion. It reveals a fascinating link to topology and the mathematics of differential geometry.

What makes this more than a quirky way to re-consider an old topic is that this perspective runs deeply through virtually all of physics, from topological states of quantum matter to features of the large-scale dynamics of the oceans and atmosphere. All of this is discussed concisely in a recent review by Pierre Delplace and Antoine Venaille (Delplace, P. \& Venaille, A. Preprint at https://arxiv.org/abs/2006.08488; 2020).

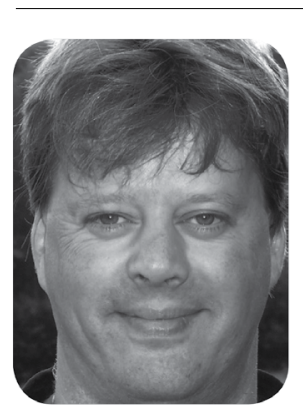

Topology plays a
unifying role in
many phenomena,
linking apparently
abstract principles
in quantum physics
with utterly
mundane effects in
daily oceanic flows.

The basic conceptual issue focuses on finding the effects created when some system - a pendulum, perhaps, or some quantum system - undergoes gradual changes in a space of defining parameters. Only in the early 1980s was this understood in a generic way for quantum theory, where the eigenstates can acquire a geometrical phase - the Berry phase, after physicist Michael Berry - due to a slow cyclic variation of parameters in the Hamiltonian. Mathematically, the situation is nearly identical to the Foucault pendulum. In that case, the phase change resulting from the translation of the pendulum over some arbitrary closed path on Earth can be calculated as a surface integral of the Gaussian curvature of the Earth over the interior of that path. In the quantum case, the curvature is different - it is the Berry curvature, depending on how the eigenstates change with the system parameters - but the phase again results from a surface integral over the interior of the closed path.

These integrals lead to topological invariants with startling consequences, most notably in the integer quantum Hall effect and more recent consideration of topological states of matter. The integer quantum Hall effect emerges in a two-dimensional electron gas in a magnetic field at low temperature. Here, the transverse electrical conductivity - the Hall conductivity - takes on specific quantized values with exceptional precision, the phenomenon being universal and independent of details such as the precise value of the magnetic field, sample disorder, electron mobility and so on. The topological origin of the phenomenon becomes clear from analysis of the Hall conductivity in terms of the Berry phase, where the conductivity ends up being equal to a special topological invariant, a Chern number. This implies the existence of protected excitations along the edges of a finite sample, which cannot change under continuous variation in the sample properties. More general invariants characterize toplogical insulators in three dimensions.

Less widely appreciated is how many further examples arise in fully classical systems. In the case of the integral quantum Hall effect, as Delplace and Venaille point out, the magnetic field breaks the prevailing time-reversal symmetry, setting the stage for topological effects. Similar effects happen for other reasons - primarily rotation - in classical wave systems. One striking experiment, for example, shows the consequences of rotation on mechanical waves in a lattice of parallel gyroscopes interacting through springs. In a certain part of the parameter space, perturbation at the edge of the lattice stirs up a wave that will not travel into the system interior, but simply moves clockwise around the boundary as a trapped edge state. A similarly bizarre effect occurs in a thin rotating fluid, where waves travel around the tank wall, again remaining away from the centre.

These waves, Delplace and Venaille suggest, are directly analogous to so-called coastal Kelvin waves, well known to travel along geophysical boundaries such as the coasts of lakes and continents, again the consequence of the Earth's rotation and the induced Coriolis force. Further examples of important topologically trapped modes include equatorial atmospheric and oceanic waves, which remain trapped in the equatorial zone, and travel eastward around the Earth. In this case, the equator isn't an actual boundary for the ocean or atmosphere. But it plays a similar role as an interface because the Coriolis force changes sign there.

Topology plays a unifying role in all these phenomena, linking apparently abstract principles in quantum physics with utterly mundane effects in daily oceanic flows. Delplace and Venaille also mention further examples in waves in stratified fluids and strange plasma waves. No doubt many more will emerge in the future.

\section{Mark Buchanan}

Published online: 4 August 2020

https://doi.org/10.1038/s41567-020-1001-y 